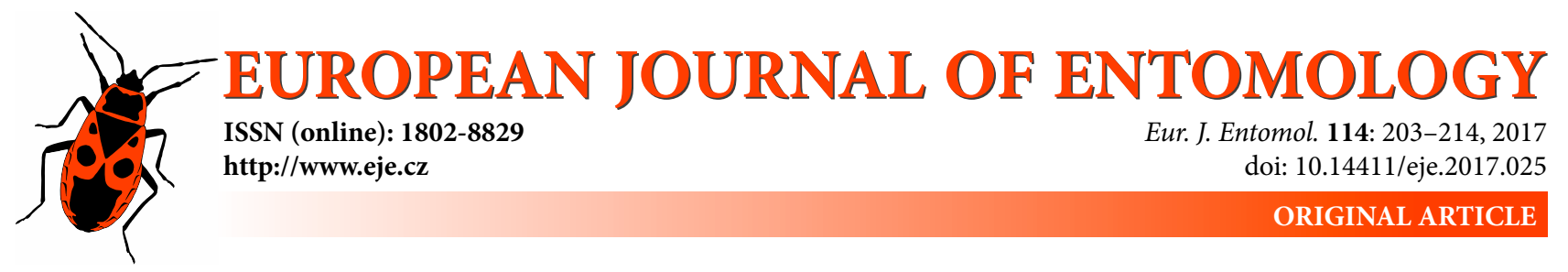

\title{
Structure of scuttle fly communities (Diptera: Phoridae) in two habitats on a Mediterranean mountain
}

\author{
CARLos GARCÍA-ROMERA and José A. BARRIENTOS
}

Departamento de Biología Animal, de Biología Vegetal y de Ecología (Unidad de Zoología), Facultad de Biociencias, Universidad Autónoma de Barcelona, E-08193 Bellaterra, Barcelona, Spain; e-mail: cgarci24@xtec.cat, JoseAntonio.Barrientos@uab.es

Key words. Diptera, Phoridae, diversity, Montseny, beech forest, scrubland

\begin{abstract}
Phoridae (scuttle flies) are widely distributed, occur in many types of habitats and are ecologically versatile, which makes them an excellent bioindicator group for evaluating faunal diversity. The structure of scuttle fly communities was compared in two Mediterranean habitats in the Montseny Natural Park (Catalonia, Spain) that differ in vegetation and microclimate: beech forest and highland scrubland. 3684 male individuals belonging to 135 species of scuttle flies were identified. Scuttle flies were more abundant in beech forest than scrubland. Observed and estimated species richness were lower in scrubland than in beech forest, while diversity was similar in both habitats. Community evenness was greater in scrubland than beech forest. Therefore, the percentage of dominant and subdominant species was higher in scrubland than beech forest, while the percentage of rare species was higher in beech forest than scrubland. Scuttle fly species composition was significantly different in the two habitats, but it was similar among plots within the same habitat. Megaselia pectoralis (Wood, 1910) and Megaselia subpleuralis (Wood, 1909) were the dominant species in beech forest, while Megaselia pusilla (Meigen, 1830), Megaselia pumila (Meigen, 1830), Megaselia superciliata (Wood, 1910) and Megaselia diversa (Wood, 1909) were the dominant species in scrubland. Trophic specialization was higher in beech forest than scrubland. Saprophages were the dominant trophic group in beech forest, while fungivores and polyphages were dominant in scrubland. The high biodiversity of scuttle flies recorded in the Montseny Natural Park indicates that there is also a high diversity of other taxa there and that these Mediterranean mountains are of high conservation status.
\end{abstract}

\section{INTRODUCTION}

Phoridae (scuttle flies) is a diverse family in the order Diptera with more than 3,700 species in more than 260 genera. Around half of the species belong to the cosmopolitan genus Megaselia Rondani. Phoridae is one of the families of Diptera with the highest diversity of larval life forms, including saprophages, predators, fungivores, parasits and parasitoids (Disney, 1990; Disney et al., 2010).

Scuttle flies are widely distributed and occur in different types of habitat, which makes them a suitable group for characterizing and comparing different ecosystems. However, few studies have compared the structure of scuttle fly communities in different habitats (Weber \& Prescher, 1990; Disney, 1994; Durska, 1996, 2001, 2006, 2013, 2015; Prescher et al., 2002; Zmihorski \& Durska, 2010) and none of them has studied them in Mediterranean mountain ecosystems. The prevalence of scuttle flies and their high biological and trophic diversity make them an excellent bioindicator group for evaluating the faunal diversity of an ecosystem as a measure of its conservation value; a high diversity of scuttle flies ussually indicates a high diversity of other taxa (Disney, 1994).
Several papers have addressed the composition of different families of Diptera in European open-area habitats (Olechowicz, 1971; Brasse, 1975; Goos, 1975; Ricou, 1978; Bährmann, 1984; Hövemeyer, 1991; Ryder et al., 2005). The scuttle fly communities in open-area habitats such as meadows, pastures, crops (Disney et al., 1981b; Weber \& Prescher, 1990, 1995; Buck, 1994, 1997; Prescher et al., 2000; Brenner, 2002, 2003, 2005, 2008) and disturbed areas in forests resulting from wildfires, clear cutting and windstorms have been studied (Durska, 2001, 2006, 2009, 2013, 2015; Prescher et al., 2002; Durska et al., 2010; Zmihorski \& Durska, 2010). However, only a few studies have surveyed the species of scuttle flies in scrublands (e.g. Disney et al., 1981a). Likewise, many papers analyse the families of Diptera in European forests, mostly in conifer-dominated forests (e.g. Bächli, 1970; Altmüller, 1976, 1979; Szadziewska, 1977; Mollon, 1982; Hövemeyer, 1984, 1991; Olechowicz, 1984, 1988), with only a few studying the species of scuttle flies (Buck, 1994, 1997; Durska, 1996, 2001, 2006, 2009, 2013, 2015; Prescher \& Haenni, 2001; Brenner, 2002, 2008; Prescher et al., 2002; Weber et al., 2006; Durska et al., 2010; Bonet et al., 2011). In contrast, studies of scuttle flies in beech 
forests or in other types of broadleaf deciduous forests are scarce (e.g. Weber \& Schiegg, 2001; Brenner, 2004; Scherber et al., 2014; Disney, 2015).

Species richness is frequently correlated with habitat structural heterogeneity. Niche specialization increases in mature and stable ecosystems and consequently saprophagous organisms are very abundant in these ecosystems due to accumulation of high quantities of organic matter (Putman, 1994). However, the diversity and species richness usually decreases with altitude and in environments with extreme abiotic factors (Begon et al., 2008).

The objective of this paper was to assess the differences in scuttle fly community structure in two ecologically contrasting habitats on a sub-humid Mediterranean mountain, beech forest and highland scrubland. Furthermore, the scuttle fly species composition in three beech forest plots that differed in soil humidity was compared and also in two scrubland plots that differed in plant composition. The hypotheses tested were that the abundance, species richness and dominance is higher in beech forest than in scrubland, which is a more unstable and extreme environment than beech forest, and that the evenness in species and trophic group abundance is greater in scrubland than in beech forest. The global scuttle fly data and the new species described in the study area is reported in García-Romera \& Barrientos (2014a) and García-Romera \& Barrientos (2014b), respectively.

\section{MATERIAL AND METHODS}

\section{Study area}

This study was carried out in the Montseny Natural Park (Catalonia, Spain), which has a high biodiversity as it contains biota of three Western European biogeographic areas: Mediterranean, Central European and Boreoalpina. The variety of mesoclimates, ecosystems and species makes the Montseny a priority conservation site, which was included in the catalogue of UNESCO biosphere reserves in 1978. The Natural Park has an area of about $400 \mathrm{~km}^{2}$; its highest mountain (Turó de l'Home) reaches 1,712 $\mathrm{m}$ a.s.l. The dominant plant communities are forests of holm oak (Quercus ilex L.) (up to $900 \mathrm{~m}$ ), beech (Fagus sylvatica L.) (from 1,000-1,500 m) and scrublands of Juniperus communis L. and Calluna vulgaris L. (above 1,500 m) (Boada \& Ullastres, 1998; Boada, 2001). The great variety of mesoclimates and ecosystems explains the high species richness of Diptera ( $>500$ species) in the Montseny Natural Park. Carles-Tolrà (1995) estimates that known species are only around $1 / 3$ of the potential number of species.

This study is based on material collected in five plots each of about $1 \mathrm{ha}$, three in beech forest and two in highland scrubland (Fig. 1). The three plots in beech forest were randomly selected and differed in soil moisture; they belong to the acidophile beech forest (ass. Luzulo Niveae-Fagetum), which is the most common type of beech forest in Montseny. The mesohumid beech forest plot (Mbf) (UTM 31TDG530274, 1,130 m a.s.1.) has deep soil and a seasonal stream flows after rain and snow melt. It is oriented W-NW with a slope of $20 \%$. The humid beech forest plot (Hbf) (UTM 31TDG532273, 1,170 m a.s.1.) has a permanent stream that sometimes floods the soil, which has a thick layer of humus and litter. It is oriented $\mathrm{N}-\mathrm{W}$, with a slope that ranges between $20-40 \%$ and is situated $570 \mathrm{~m}$ from Mbf. The dry beech forest plot (Dbf) (UTM 31TDG540279, 1,250 m a.s.1.) is far from any

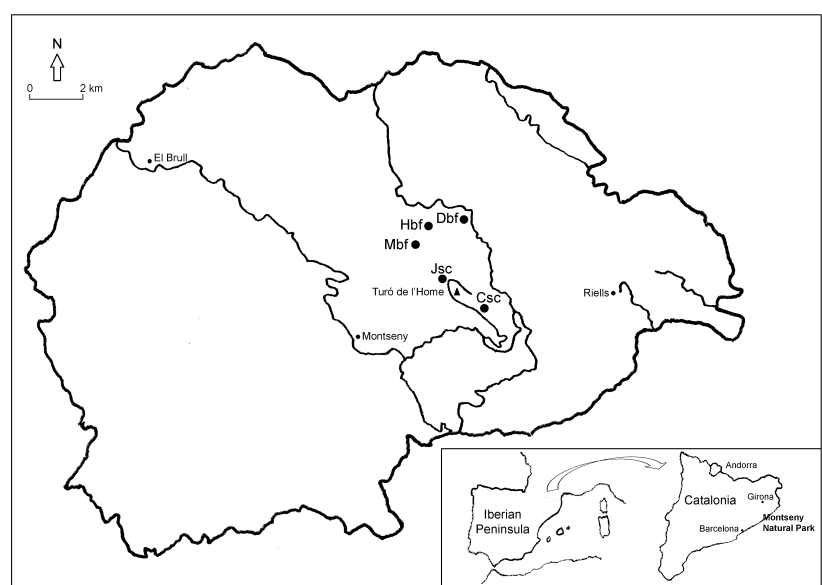

Fig. 1. Map showing the location of the study plots in Montseny Natural Park and inset maps of Iberian Peninsula and Catalonia showing the location of the Park. Mbf - mesohumid beech forest, $\mathrm{Hbf}$ - humid beech forest, Dbf - dry beech forest, Jsc - Juniperus scrubland, Csc - Calluna scrubland.

stream and has shallow soil. Beech growth is significantly lower than in the other beech plots and there is a dense herbaceous plant layer. It is oriented to the $\mathrm{N}$, with a slope of $10 \%$ and is situated $960 \mathrm{~m}$ from Hbf.

The two highland plots were randomly selected and differ in their plant composition. Near the mountain summit, we sampled a Juniperus communis L. scrubland (ass. Juniperion nanae) plot (Jsc) (UTM 31TDG530256, 1,620 m a.s.1.). It is oriented ENE, on a steep slope and is situated 1,540 m from Mbf. At a lower altitude, we sampled a Calluna vulgaris (L.) scrubland (ass. Violocaninae-Callunetum) plot (Csc) (UTM 31TDG542245, 1,510 m a.s.1.) on a windy site. It is oriented to the $\mathrm{N}$, with a slight slope and is situated $1,770 \mathrm{~m}$ from Jsc.

\section{Sampling methods}

Sampling was carried out from March 1990 to March 1991. The first week we sampled the beech forest plots and the following week the two scrubland plots.

The same effort was made to sample each of the plots using the following methods:

\section{Pitfall traps}

Four traps, $9 \mathrm{~cm}$ in diameter, arranged in line, $2 \mathrm{~m}$ apart, were placed in each plot. Each trap contained $4 \%$ formaldehyde with an antifreeze liquid in winter.

\section{Window traps}

We used one trap per plot. It had a transparent glass of $0.80 \times$ $0.40 \mathrm{~m}$ (width $\times$ height), with a collector plate on each side containing $4 \%$ formaldehyde and detergent. Each trap was anchored to the soil.

\section{Emergence traps}

A metallic cylinder (50 cm in diameter) attached to a cone with a boat collector at the top containing $70 \%$ ethanol. Two traps were placed in each plot and randomly changed every fortnight.

All traps were permanently left in the field during the sampling year and were emptied every fortnight.

\section{Data analysis}

The material was preserved in $70 \%$ ethanol. Analyses were only based on males, as most females of the genus Megaselia Rondani and Phora Latreille are not currently identifiable to species level. 
We used relative abundance $\left(A_{r}\right)$ to determine the dominance in community structure:

$$
A_{r}=\frac{n_{i}}{N} \cdot 100
$$

where $n_{i}=$ number of individuals of $i$ species in the sample and $N=$ number of individuals of the scuttle fly community in the sample.

The degree of dominance was classified into five categories according to species $A_{r}$, based on Durska (1981, 2001): Eudominant: over $15.0 \%$, Dominant: from 5.1 to $15.0 \%$, Subdominant: from 2.1 to $5.0 \%$, Influential: from 1.1 to $2.0 \%$ and Accessory (rare): up to $1.0 \%$.

Species diversity was quantified using the Shannon index $\left(H^{\prime}\right)$ :

$$
H^{\prime}=-\sum p_{i} \cdot \ln \cdot p_{i}
$$

where $p_{i}=n_{i} / N$ (relative abundance).

Shannon evenness $\left(J^{\prime}\right)$, which considers the degree of evenness in species abundances (Moreno, 2001; Magurran, 2004)

$$
J^{\prime}=\frac{H^{\prime}}{H_{\max }}
$$

where $H_{\max }=\ln S$ and $S$ is the number of species in the sample.

Species accumulation curves were used to determine the estimated number of species in each plot. This curve is the cumulative number of species recorded as a function of the cumulative number of samples (sampling effort) (Colwell et al., 2004). Twenty-one fortnightly samples were used in this analysis. Species accumulation curves and their estimators were implemented in EstimateS software (Colwell, 2009). The non-parametric estimators Chao 1, Chao 2, ACE, ICE, Jackknife 1, Jackknife 2, Bootstrap were used; these are described in Colwell \& Coddington (1994) and Chazdon et al. (1998). These estimators allow to know the percentage of species that were captured related to those estimated, i.e., the efficiency of the sampling effort.

\section{Statistical analysis}

Pearson's correlation analysis (Sokal \& Rohlf, 1995) was used to compare trophic groups in the two habitats. Normality was checked using the Kolmogorov-Smirnov goodness of fit test ( $\alpha=$ 0.05). Statistical analyses were performed using SPSS 15.0 software for Windows (Chicago, SPSS Inc.).

Agglomerative Hierarchical Cluster analysis with averagelinkage clustering and dendrograms were used to group the plots, as a function of scuttle flies species composition. We used BrayCurtis similarity index for quantitative data, using square-root transformed data. A similarity test profile (SIMPROF) was used to assess if plots within a cluster differed significantly. A SIMPER (similarity percentages) analysis was used to determine the species that contribute most to the similarity among plots in the same habitat and those that differentiate them. These tests were performed using PRIMER 6 software (Clarke \& Gorley, 2006).

Non-parametric PERMANOVA (permutational ANOVA) with repeated measures (for fortnightly samples) design on Euclidean distance was done to compare density, species richness and diversity of the scuttle fly community in the two habitats studied, based on a two-factor design, habitat (fixed) and time (random), with data for the five plots. A PERMDISP routine was used to test the homogeneity of dispersion between habitats. Permutational analysis does not assume homogeneity of variances or that the errors are independent and normally distributed, but that the samples are independent and with homogeneity of dispersion. However, we consider that a non-significant result from PERMDISP $(p>0.05)$ is not strictly necessary prior to using PERMANOVA. Permutational MANOVA based on Bray-Curtis similarity was used to determine whether there was significant difference in spe- cies composition between habitats. This test does not assume a multivariate normal distribution, unrealistic for most ecological data sets (Anderson, 2001; Anderson et al., 2008). A distance matrix between observations was calculated (with prior square root data transformation) from a raw multivariate matrix, where the variables are scuttle fly species (rows) and fortnightly samples for the five plots (columns). In both permutational tests the statistical pseudo-F and p-value were calculated after 9,999 permutations. Significant level was set at $\alpha=0.05$. Tests were carried out using the PERMANOVA + for PRIMER software (Anderson et al., 2008).

\section{RESULTS}

\section{Dominance structure of the scuttle fly communities}

We captured a total of 95,330 Diptera of which 7,045 were scuttle flies $(7.39 \%)$. Scuttle flies were more abundant in the beech forest and made up a higher percentage of the Diptera (5,846 Phoridae out of 72,004 Diptera, 8.11\%) than in scrubland (1,199 Phoridae out of 23,326 Diptera, $5.14 \%)$.

We identified 3,684 scuttle fly males belonging to 135 species, of which 107 species belong to the genus Megaselia Rondani $(79.3 \%)$, with a relative abundance of $88.8 \%$. In all plots, we found a few dominant and eudominant species, while most were accessory species (Table 1). Megaselia pectoralis was the only eudominant species in the three beech forest plots while this species was subdominant in the scrubland plots. M. pectorella was dominant in Mbf, subdominant in $\mathrm{Hbf}$, and absent in scrubland plots. $M$. diversa was a dominant species in Mbf and in both scrubland plots. M. pumila and M. pusilla were only dominant in scrubland plots; $M$. pusilla was also eudominant in Jsc. M. subpleuralis had a higher relative abundance in Dbf than in Hbf. M. superciliata was dominant in scrubland and in Dbf. Metopina galeata had a higher relative abundance in scrubland than in beech forest. Several species dominated in one plot but were accessory in the remaining plots. For instance, Tripheba opaca and Megaselia brevicostalis were only dominant in Csc, similarly Diplonevra florescens was only dominant in $\mathrm{Hbf}$.

We found nine characteristic species of beech forest (Fig. 2a), with one eudominant species, Megaselia pectoralis, one dominant species, $M$. subpleuralis and seven subdominant species: $M$. pusilla, $M$. diversa, $M$. superciliata, $M$. pectorella, Diplonevra florescens, $M$. longicostalis and $M$. basispinata. The relative abundance of all these species was $67.9 \%$ (Table 2). We found twelve characteristic species of scrubland (Fig. 2b), with one eudominant species, M. pusilla, three dominant species, M. pumila, M. superciliata and $M$. diversa, and nine subdominant: $M$. brevicostalis, Diplonevra nitidula, M. basispinata, M. longicostalis, M. largifrontalis, Metopina galeata, Megaselia pectoralis, Triphleba opaca and Megaselia xanthozona. The relative abundance of all these species accounted for $77.8 \%$. The percentage of accessory species was higher in beech forest than in scrubland (Table 2), while middle categories (subdominants and influential) were more abundant in scrubland than beech forest. Common species in both habitats 
Table 1. Species composition with the number of individuals captured $(I n d)$ and relative abundance $\left(A_{r}\right)$ of the different species in the scuttle fly communities in three beech forest plots and two scrubland plots in the Montseny Natural Park. tg - trophic groups, $f-$ fungivores, p - polyphages, s - saprophages, z - zoophages (García-Romera \& Barrientos, 2014a). Mbf - mesohumid beech forest, Hbf - humid beech forest, Dbf - dry beech forest, Jsc - Juniperus scrubland, Csc - Calluna scrubland.

\begin{tabular}{|c|c|c|c|c|c|c|c|c|c|c|c|}
\hline \multirow{2}{*}{ Species } & \multirow{2}{*}{$\operatorname{tg}$} & \multicolumn{2}{|c|}{ Mbf } & \multicolumn{2}{|c|}{$\mathrm{Hbf}$} & \multicolumn{2}{|c|}{ Dbf } & & & & \\
\hline & & Ind & $A_{r}$ & Ind & $A_{r}$ & Ind & $A_{r}$ & Ind & $A_{r}$ & Ind & $A_{r}$ \\
\hline Anevrina thoracica (Meigen, 1804) & $\mathrm{s}$ & 3 & 0.41 & 3 & 0.36 & & & & & & \\
\hline Anevrina unispinosa (Zetterstedt, 1860) & $\mathrm{s}$ & & & 1 & 0.12 & 1 & 0.07 & & & & \\
\hline Borophaga femorata (Meigen, 1830) & & 20 & 2.74 & 7 & 0.83 & 18 & 1.31 & 6 & 1.44 & 2 & 0.63 \\
\hline Conicera dauci (Meigen, 1830) & $p$ & 2 & 0.27 & 1 & 0.12 & & & 1 & 0.24 & & \\
\hline Conicera floricola Schmitz, 1938 & S & 2 & 0.27 & 12 & 1.42 & & & & & & \\
\hline Conicera similis (Haliday, 1833) & $\mathrm{p}$ & 1 & 0.14 & 2 & 0.24 & & & & & & \\
\hline Conicera tarsalis Schmitz, 1920 & S & 1 & 0.14 & 3 & 0.36 & 1 & 0.07 & & & & \\
\hline Conicera tibialis Schmitz, 1925 & $\mathrm{~s}$ & & & & & 1 & 0.07 & & & & \\
\hline Diplonevra florescens (Tuton, 1801) & $\mathrm{s}$ & 11 & 1.51 & 88 & 10.44 & 5 & 0.36 & & & 1 & 0.32 \\
\hline Diplonevra funebris (Meigen, 1830) & $\mathrm{s}$ & & & & & & & 2 & 0.48 & 3 & 0.95 \\
\hline Diplonevra glabra (Schmitz, 1927) & & & & & & & & 3 & 0.72 & & \\
\hline Diplonevra nitidula (Meigen, 1830) & z & 7 & 0.96 & 29 & 3.44 & 2 & 0.15 & 26 & 6.22 & 5 & 1.58 \\
\hline Diplonevra pachycera (Schmitz, 1918) & & 6 & 0.82 & 16 & 1.90 & 1 & 0.07 & & & & \\
\hline Diplonevra unisetalis (Schmitz, 1935) & & & & & & & & 2 & 0.48 & 5 & 1.58 \\
\hline Gymnophora arcuata (Meigen, 1830) & s & 1 & 0.14 & & & & & & & & \\
\hline Megaselia aculeata (Schmitz, 1919) & & 3 & 0.41 & 8 & 0.95 & & & & & & \\
\hline Megaselia aequalis (Wood, 1909) & & 2 & 0.27 & 4 & 0.47 & 1 & 0.07 & & & & \\
\hline Megaselia albicans (Wood, 1908) & $\mathrm{f}$ & 1 & 0.14 & & & & & & & & \\
\hline Megaselia albicaudata (Wood, 1910) & & & & 1 & 0.12 & & & & & 1 & 0.32 \\
\hline Megaselia altifrons (Wood, 1909) & & 1 & 0.14 & & & 2 & 0.15 & & & & \\
\hline Megaselia angusta (Wood, 1909) & $p$ & 25 & 3.42 & 19 & 2.25 & 6 & 0.44 & 10 & 2.39 & 2 & 0.63 \\
\hline Megaselia angustiata Schmitz, 1936 & & & & & & & & & & 1 & 0.32 \\
\hline Megaselia annulipes (Schmitz, 1921) & z & & & 1 & 0.12 & & & & & & \\
\hline Megaselia arbuciensis García-Romera, 2014 & & & & & & 2 & 0.15 & & & & \\
\hline Megaselia barrientosi García-Romera, 2014 & & 1 & 0.14 & & & & & & & & \\
\hline Megaselia basispinata (Lundbeck, 1920) & & 27 & 3.70 & 11 & 1.30 & 31 & 2.25 & 14 & 3.35 & 12 & 3.79 \\
\hline Megaselia berndseni (Schmitz, 1919) & $f$ & 1 & 0.14 & 1 & 0.12 & & & 1 & 0.24 & 1 & 0.32 \\
\hline Megaselia bifurcata Disney, 1983 & & 1 & 0.14 & & & & & & & & \\
\hline Megaselia bovista (Gimmerthal, 1848) & $f$ & & & & & & & & & 1 & 0.32 \\
\hline Megaselia brevicostalis (Wood, 1910) & $\mathrm{s}$ & 2 & 0.27 & 1 & 0.12 & 1 & 0.07 & 15 & 3.59 & 21 & 6.62 \\
\hline Megaselia brevior (Schmitz, 1924) & $\mathrm{s}$ & & & 2 & 0.24 & & & & & & \\
\hline Megaselia brunnea (Schmitz, 1920) & & & & & & & & & & 1 & 0.32 \\
\hline Megaselia callunae García-Romera, 2014 & & & & 1 & 0.12 & & & & & 1 & 0.32 \\
\hline Megaselia campestris (Wood, 1908) & & & & & & 1 & 0.07 & & & & \\
\hline Megaselia cinereifrons (Strobl, 1910) & $f$ & 1 & 0.14 & & & 4 & 0.29 & & & & \\
\hline Megaselia clemonsi Disney, 1984 & & & & & & 1 & 0.07 & & & 1 & 0.32 \\
\hline Megaselia coaetanea Schmitz, 1929 & $f$ & & & 1 & 0.12 & & & 3 & 0.72 & 2 & 0.63 \\
\hline Megaselia collini (Wood, 1909) & & & & 1 & 0.12 & & & & & & \\
\hline Megaselia consetigera (Schmitz, 1925) & & & & 2 & 0.24 & & & & & & \\
\hline Megaselia costalis (Von Roser, 1840) & & & & 1 & 0.12 & & & & & & \\
\hline Megaselia crassipes (Wood, 1909) & & 1 & 0.14 & & & 4 & 0.29 & & & & \\
\hline Megaselia curvicapilla Schmitz, 1947 & & & & 16 & 1.90 & 4 & 0.29 & 1 & 0.24 & & \\
\hline Megaselia dahli (Becker, 1901) & & 1 & 0.14 & 7 & 0.83 & & & 3 & 0.72 & 2 & 0.63 \\
\hline Megaselia discreta (Wood, 1909) & $f$ & 2 & 0.27 & & & 2 & 0.15 & & & & \\
\hline Megaselia diversa (Wood, 1909) & $\mathrm{s}$ & 40 & 5.48 & 31 & 3.68 & 41 & 2.98 & 30 & 7.18 & 25 & 7.89 \\
\hline Megaselia elongata (Wood, 1914) & z & & & 1 & 0.12 & & & & & 1 & 0.32 \\
\hline Megaselia erecta (Wood, 1910) & & & & & & 1 & 0.07 & & & & \\
\hline Megaselia eupygis Schmitz, 1929 & & & & & & 1 & 0.07 & & & & \\
\hline Megaselia flava (Fallén, 1823) & $f$ & 1 & 0.14 & 1 & 0.12 & 2 & 0.15 & & & & \\
\hline Megaselia flavicans Schmitz, 1935 & $f$ & 16 & 2.19 & 25 & 2.97 & 16 & 1.16 & & & 1 & 0.32 \\
\hline Megaselia frameata Schmitz, 1927 & f & 2 & 0.27 & & & & & & & & \\
\hline Megaselia fumata (Malloch, 1909) & & 6 & 0.82 & & & 2 & 0.15 & & & & \\
\hline Megaselia fuscovariana Schmitz, 1933 & & 6 & 0.82 & 2 & 0.24 & & & & & & \\
\hline Megaselia giraudii (Egger, 1862) & $p$ & 8 & 1.10 & 6 & 0.71 & 1 & 0.07 & & & & \\
\hline Megaselia glabrifrons (Wood, 1909) & & 2 & 0.27 & & & 3 & 0.22 & 1 & 0.24 & & \\
\hline Megaselia hirsuta (Wood, 1910) & & 1 & 0.14 & 1 & 0.12 & & & & & & \\
\hline Megaselia hirtiventris (Wood, 1909) & $f$ & & & & & & & & & 1 & 0.32 \\
\hline Megaselia iberiensis Disney, 1999 & & & & & & & & 1 & 0.24 & 1 & 0.32 \\
\hline Megaselia indifferens (Lundbeck, 1920) & & & & & & 1 & 0.07 & & & & \\
\hline Megaselia infraposita (Wood, 1909) & & 2 & 0.27 & 4 & 0.47 & 6 & 0.44 & & & & \\
\hline Megaselia introlapsa Schmitz, 1937 & & 11 & 1.51 & 8 & 0.95 & 2 & 0.15 & 1 & 0.24 & & \\
\hline Megaselia involuta (Wood, 1910) & & 16 & 2.19 & 2 & 0.24 & 6 & 0.44 & & & & \\
\hline Megaselia ivanis García-Romera, 2014 & & 1 & 0.14 & 1 & 0.12 & 3 & 0.22 & & & & \\
\hline Megaselia lactipennis (Lundbeck, 1920) & & & & & & & & 6 & 1.44 & 5 & 1.58 \\
\hline Megaselia largifrontalis Schmitz, 1939 & & 6 & 0.82 & 3 & 0.36 & 4 & 0.29 & 8 & 1.91 & 17 & 5.36 \\
\hline Megaselia lata (Wood, 1910) & $f$ & 29 & 3.97 & 11 & 1.30 & 17 & 1.24 & & & & \\
\hline Megaselia latifrons (Wood, 1910) & & & & 4 & 0.47 & & & 1 & 0.24 & & \\
\hline Megaselia latior Schmitz, 1936 & $f$ & 2 & 0.27 & & & & & & & & \\
\hline Megaselia longicostalis (Von Roser, 1840) & $\mathrm{p}$ & 18 & 2.47 & 12 & 1.42 & 42 & 3.05 & 15 & 3.59 & 10 & 3.15 \\
\hline Megaselia longifurca (Lundbeck, 1921) & Z & & & 4 & 0.47 & 1 & 0.07 & & & & \\
\hline Megaselia longipalpis (Wood, 1910) & & & & 1 & 0.12 & 3 & 0.22 & & & & \\
\hline Megaselia lutea (Meigen, 1830) & $f$ & 1 & 0.14 & 2 & 0.24 & 3 & 0.22 & & & 1 & 0.32 \\
\hline Megaselia malhamensis Disney, 1986 & & & & 1 & 0.12 & & & & & & \\
\hline Megaselia mallochi (Wood, 1909) & Z & & & & & 1 & 0.07 & 13 & 3.11 & 1 & 0.32 \\
\hline
\end{tabular}


Table 1 (continued).

\begin{tabular}{|c|c|c|c|c|c|c|c|c|c|c|c|}
\hline \multirow{2}{*}{ Species } & \multirow{2}{*}{$\operatorname{tg}$} & \multicolumn{2}{|c|}{ Mbf } & \multicolumn{2}{|c|}{$\mathrm{Hbf}$} & \multicolumn{2}{|c|}{ Dbf } & \multicolumn{2}{|c|}{ Jsc } & \multicolumn{2}{|c|}{ Csc } \\
\hline & & Ind & $A_{r}$ & Ind & $A_{r}$ & Ind & $A_{r}$ & Ind & $A_{r}$ & Ind & $A_{r}$ \\
\hline Megaselia manicata (Wood, 1910) & & & & & & 1 & 0.07 & 2 & 0.48 & & \\
\hline Megaselia marekdurskii Disney, 1998 & & & & 3 & 0.36 & 3 & 0.22 & & & & \\
\hline Megaselia meconicera (Speiser, 1925) & $\mathrm{s}$ & 1 & 0.14 & & & & & & & & \\
\hline Megaselia minuta (Aldrich, 1892) & $\mathrm{s}$ & & & & & & & 1 & 0.24 & 1 & 0.32 \\
\hline Megaselia monochaeta Strobl, 1892 & & 11 & 1.51 & 17 & 2.02 & 16 & 1.16 & 2 & 0.48 & 4 & 1.26 \\
\hline Megaselia montseniensis García-Romera, 2014 & & & & 1 & 0.12 & & & & & & \\
\hline Megaselia nectergata Disney, 1999 & & 1 & 0.14 & 3 & 0.36 & 1 & 0.07 & 9 & 2.15 & 5 & 1.58 \\
\hline Megaselia nigra (Meigen, 1830) & $p$ & 3 & 0.41 & & & 15 & 1.09 & 1 & 0.24 & 1 & 0.32 \\
\hline Megaselia palmeni (Becker, 1901) & & & & 2 & 0.24 & & & & & 2 & 0.63 \\
\hline Megaselia pectoralis (Wood, 1910) & $\mathrm{s}$ & 204 & 27.95 & 278 & 32.98 & 423 & 30.74 & 16 & 3.83 & 8 & 2.52 \\
\hline Megaselia pectorella Schmitz, 1929 & $\mathrm{~s}$ & 40 & 5.48 & 39 & 4.63 & 26 & 1.89 & & & & \\
\hline Megaselia pedatella (Schmitz, 1926) & & 3 & 0.41 & 4 & 0.47 & 17 & 1.24 & 1 & 0.24 & & \\
\hline Megaselia pleuralis (Wood, 1909) & $p$ & 3 & 0.41 & 8 & 0.95 & 13 & 0.94 & 2 & 0.48 & & \\
\hline Megaselia plurispinulosa (Zetterstedt, 1860) & f & & & 1 & 0.12 & & & & & & \\
\hline Megaselia propinqua (Wood, 1909) & & & & & & & & 1 & 0.24 & & \\
\hline Megaselia pulicaria (Fallén, 1823) & $\mathrm{s}$ & 4 & 0.55 & & & 5 & 0.36 & 1 & 0.24 & & \\
\hline Megaselia pumila (Meigen, 1830) & $f$ & 3 & 0.41 & 7 & 0.83 & 2 & 0.15 & 55 & 13.16 & 46 & 14.51 \\
\hline Megaselia pusilla (Meigen, 1830) & $\mathrm{p}$ & 34 & 4.66 & 38 & 4.51 & 46 & 3.34 & 85 & 20.33 & 47 & 14.83 \\
\hline Megaselia rubella (Schmitz, 1920) & f & 1 & 0.14 & 3 & 0.36 & 5 & 0.36 & 1 & 0.24 & & \\
\hline Megaselia rufa (Wood, 1908) & $z$ & & & 1 & 0.12 & & & & & & \\
\hline Megaselia ruficornis (Meigen, 1830) & $\mathrm{s}$ & 4 & 0.55 & 6 & 0.71 & 3 & 0.22 & & & & \\
\hline Megaselia rufifrons (Wood, 1910) & $\mathrm{s}$ & 1 & 0.14 & & & 1 & 0.07 & & & & \\
\hline Megaselia rufipes (Meigen, 1804) & $\mathrm{p}$ & 4 & 0.55 & 1 & 0.12 & 2 & 0.15 & 1 & 0.24 & & \\
\hline Megaselia rupestris Schmitz, 1934 & & & & & & 1 & 0.07 & & & & \\
\hline Megaselia sarae García-Romera, 2014 & & 1 & 0.14 & & & & & & & & \\
\hline Megaselia scutellaris (Wood, 1909) & $f$ & 3 & 0.41 & 5 & 0.59 & 26 & 1.89 & 3 & 0.72 & 2 & 0.63 \\
\hline Megaselia sericata Schmitz, 1935 & & 4 & 0.55 & & & & & & & & \\
\hline Megaselia setulipalpis Schmitz, 1938 & & & & 1 & 0.12 & & & & & & \\
\hline Megaselia simulans (Wood, 1912) & & 1 & 0.14 & 1 & 0.12 & & & & & & \\
\hline Megaselia sp1 & & 1 & 0.14 & 2 & 0.24 & & & 1 & 0.24 & & \\
\hline Megaselia spinicincta (Wood, 1910) & $f$ & 2 & 0.27 & & & & & & & & \\
\hline Megaselia spinigera (Wood, 1908) & & & & 1 & 0.12 & & & & & & \\
\hline Megaselia stichata (Lundbeck, 1920) & & & & & & 1 & 0.07 & & & 1 & 0.32 \\
\hline Megaselia stigmatica (Schmitz, 1920) & & & & & & & & & & 1 & 0.32 \\
\hline Megaselia striolata Schmitz, 1940 & & & & & & & & 3 & 0.72 & 2 & 0.63 \\
\hline Megaselia subconvexa (Lundbeck, 1920) & & 1 & 0.14 & & & & & & & & \\
\hline Megaselia subfraudulenta Schmitz, 1933 & & 3 & 0.41 & 3 & 0.36 & 12 & 0.87 & & & & \\
\hline Megaselia subfuscipes Schmitz, 1935 & $\mathrm{~s}$ & 13 & 1.78 & 2 & 0.24 & 3 & 0.22 & 7 & 1.67 & & \\
\hline Megaselia subpalpalis (Lundbeck, 1920) & & & & & & 1 & 0.07 & & & & \\
\hline Megaselia subpleuralis (Wood, 1909) & & 48 & 6.58 & 19 & 2.25 & 345 & 25.07 & 1 & 0.24 & 2 & 0.63 \\
\hline Megaselia subtumida (Wood, 1909) & $\mathrm{s}$ & 9 & 1.23 & 8 & 0.95 & 4 & 0.29 & & & & \\
\hline Megaselia superciliata (Wood, 1910) & & 8 & 1.10 & 4 & 0.47 & 94 & 6.83 & 31 & 7.42 & 24 & 7.57 \\
\hline Megaselia sylvatica (Wood, 1910) & $f$ & 2 & 0.27 & & & 1 & 0.07 & & & & \\
\hline Megaselia tarsalis (Wood, 1910) & s & 3 & 0.41 & 1 & 0.12 & 5 & 0.36 & 1 & 0.24 & & \\
\hline Megaselia teneripes Schmitz, 1957 & & 1 & 0.14 & & & & & & & & \\
\hline Megaselia variana Schmitz, 1926 & & 8 & 1.10 & 8 & 0.95 & 11 & 0.80 & & & 1 & 0.32 \\
\hline Megaselia vernalis (Wood, 1909) & & 3 & 0.41 & 5 & 0.59 & 12 & 0.87 & & & 4 & 1.26 \\
\hline Megaselia xanthozona (Strobl, 1892) & & & & & & 1 & 0.07 & 5 & 1.20 & 12 & 3.79 \\
\hline Metopina braueri (Strobl, 1880) & & & & 1 & 0.12 & & & & & & \\
\hline Metopina galeata (Haliday, 1833) & $\mathrm{s}$ & 2 & 0.27 & & & 2 & 0.15 & 18 & 4.31 & 6 & 1.89 \\
\hline Metopina heselhausi Schmitz, 1914 & $\mathrm{~s}$ & & & & & & & 3 & 0.72 & 1 & 0.32 \\
\hline Phora atra (Meigen, 1804) & $\mathrm{s}$ & 1 & 0.14 & & & & & & & 1 & 0.32 \\
\hline Triphleba beatricis García-Romera, 2014 & & 2 & 0.27 & & & & & & & & \\
\hline Triphleba distinguenda (Strobl, 1892) & $\mathrm{s}$ & 3 & 0.41 & 6 & 0.71 & & & & & & \\
\hline Triphleba inaequalis Schmitz, 1943 & & 2 & 0.27 & & & 6 & 0.44 & & & & \\
\hline Triphleba intempesta (Schmitz, 1918) & & 1 & 0.14 & & & 15 & 1.09 & & & 1 & 0.32 \\
\hline Triphleba intermedia (Malloch, 1908) & & & & & & 1 & 0.07 & 1 & 0.24 & 1 & 0.32 \\
\hline Triphleba opaca (Meigen, 1830) & s & & & & & 6 & 0.44 & 3 & 0.72 & 18 & 5.68 \\
\hline Triphleba papillata (Wingate, 1906) & $\mathrm{s}$ & & & 3 & 0.36 & 1 & 0.07 & & & 1 & 0.32 \\
\hline Triphleba trinervis (Becker, 1901) & $\mathrm{f}$ & & & & & 2 & 0.15 & & & & \\
\hline Triphleba ypsilon Carles-Tolrá \& García-Romera, 2011 & & & & 2 & 0.24 & 3 & 0.22 & & & & \\
\hline
\end{tabular}

were M. pectoralis, M. diversa, M. pusilla, M. basispinata, M. superciliata and M. longicostalis.

\section{Trophic groups}

Larval habit is known for only about $50 \%$ of the species collected. Saprophagous species made up the highest number of species in all plots, followed by fungivorous and the polyphagous species. The percentage of species in the different trophic groups in beech forest and scrubland was similar. The number of species in each trophic group in both habitats was highly and positively correlated $(\mathrm{r}=$
$0.99, p=0.014$ ) (Fig. 3a, b). However, in relation to number of individuals, saprophagous species were dominant in beech forest, and about as abundant as polyphagous species in scrubland (Fig. 3c, d), with no significant correlation between habitats in the number of individuals in each trophic group $(\mathrm{r}=0.70 ; \mathrm{p}=0.302)$.

\section{Density and species richness estimates}

Scuttle fly density (ind $\cdot \mathrm{m}^{-2}$. day $^{-1}$ ) measured using window traps differed significantly in the two habitats (pseudo$\mathrm{F}=6.64, \mathrm{p}=0.016$ ) although no differences were found in 


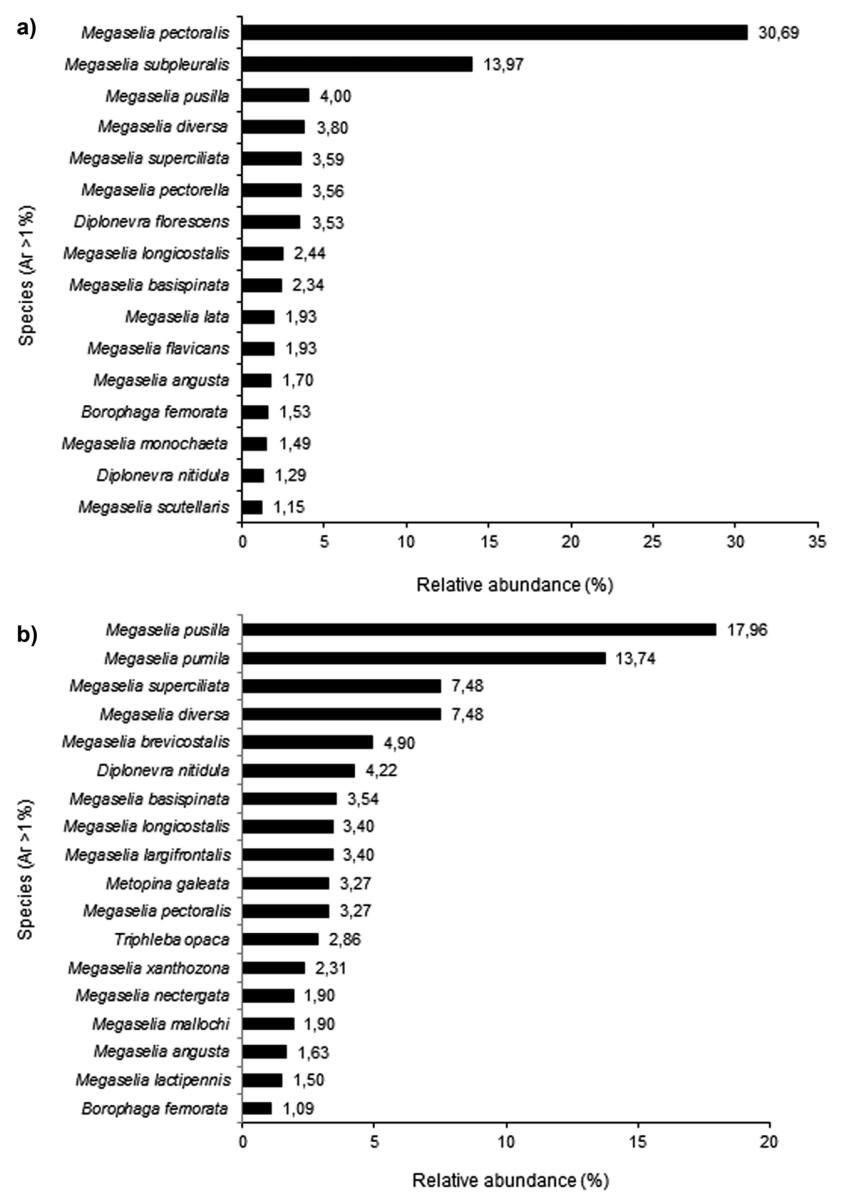

Fig. 2. Dominance structure of the scuttle fly communities in beech forest (a) and scrubland (b). Mean relative abundance of the species in plots in each habitat (abundance $>1 \%$ ).

dispersion $(p=0.0097)$. Mean density was higher in beech forest (especially in Dbf) than scrubland (Fig. 4).

Species accumulation curves for beech forest were nonasymptotic, except for Dbf and Mbf, for which the ACE curve was asymptotic or close to it, respectively (Fig. 5a, $b, c)$. The number of species recorded were similar in the three beech forest plots. However, a higher number of species was estimated for Mbf and Hbf than Dbf, and the efficiency of sampling was a little higher for Dbf than Mbf and $\mathrm{Hbf}$. The percentage of singletons was high in all plots, so a high number of species were not captured (Table 3 ).

In scrubland, none of the species accumulation curves for Jsc were asymptotic; however Chao 1 and Jack 2 were asymptotic for Csc (Fig. 5d, e). The numbers of species recorded were similar in the scrubland plots, although the number estimated for Csc was higher than for Jsc, while the efficiency of sampling effort was higher for Jsc than

Table 2. Species richness (S) and relative frequency (\%) of each dominance group in beech forest and scrubland.

\begin{tabular}{lccccc}
\hline \multirow{2}{*}{ Dominance group } & \multicolumn{2}{c}{ Beech forest } & & \multicolumn{2}{c}{ Scrubland } \\
\cline { 2 - 3 } \cline { 5 - 6 } & $\mathrm{S}$ & $\%$ & & $\mathrm{~S}$ & $\%$ \\
\hline Eudominants & 1 & 0.83 & & 1 & 1.49 \\
Dominants & 1 & 0.83 & & 3 & 4.48 \\
Subdominants & 7 & 5.79 & & 9 & 13.43 \\
Influential & 7 & 5.79 & & 5 & 7.46 \\
Accesory & 105 & 86.78 & & 49 & 73.13 \\
TOTAL & 121 & & & 67 & \\
\hline
\end{tabular}
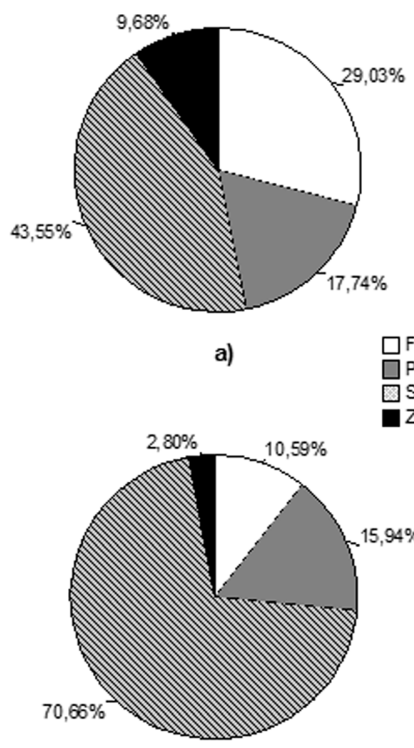

c)

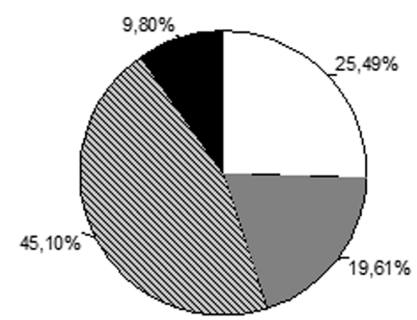

b)

$$
\begin{aligned}
& \text { Fungivores } \\
& \text { Polyphages } \\
& \text { Saprophages }
\end{aligned}
$$

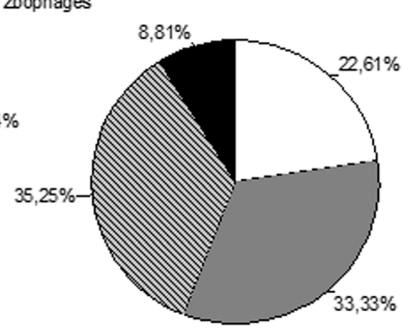

d)

Fig. 3. Percentage mean species richness of each trophic group in beech forest (a) and scrubland plots (b). Mean relative abundance of each trophic group in the beech forest (c) and scrubland plots (d).

Csc. The percentage of singletons recorded for Csc was the highest of all the plots (Table 3 ).

In summary, the observed and estimated species richness and sampling effort efficiency were higher in beech forest than scrubland.

\section{a-diversity}

Species richness was significantly higher in beech forest than in scrubland (pseudo-F $=7.54, \mathrm{p}=0.03$ ) and dispersion was homogeneous $(\mathrm{F}=3.15, \mathrm{p}=0.079)$. The Shannon index $\left(H^{\prime}\right)$ was not significantly different for the two habitats (pseudo-F $=0.267 ; \mathrm{p}=0.64$ ), but comparison of plots revealed that $H^{\prime}$ 'was lower for Dbf than the other plots. Shannon evenness $\left(J^{\prime}\right)$ differed significantly between habitats (pseudo- $\mathrm{F}=8.61, \mathrm{p}=0.02$ ), although dispersion was not homogeneous $(\mathrm{F}=20.7, \mathrm{p}<0.001)$. Evenness was

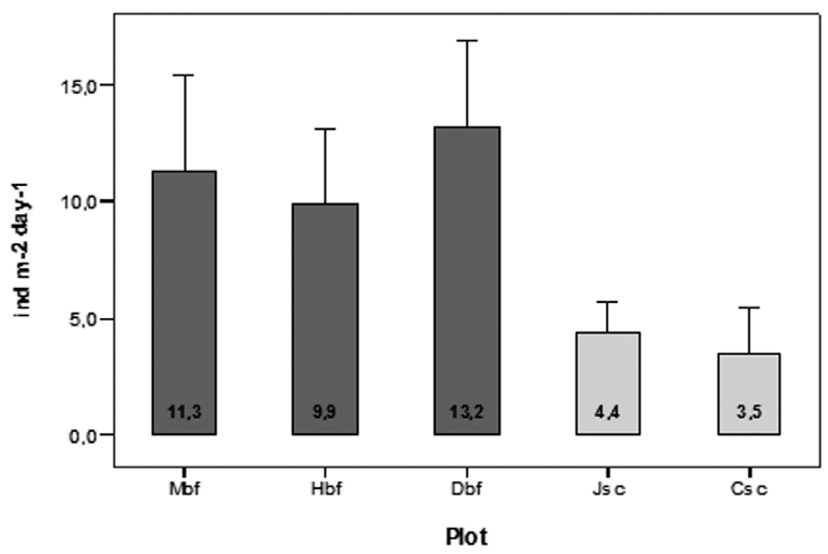

Fig. 4. Mean density (ind $\mathrm{m}^{-2}$ day $^{-1}$ ) of the scuttle flies recorded in the five plots in the two habitats. Vertical bars are standard errors. Mbf - mesohumid beech forest, Hbf - humid beech forest, Dbf - dry beech foest, Jsc - Juniperus scrubland, Csc - Calluna scrubland. 

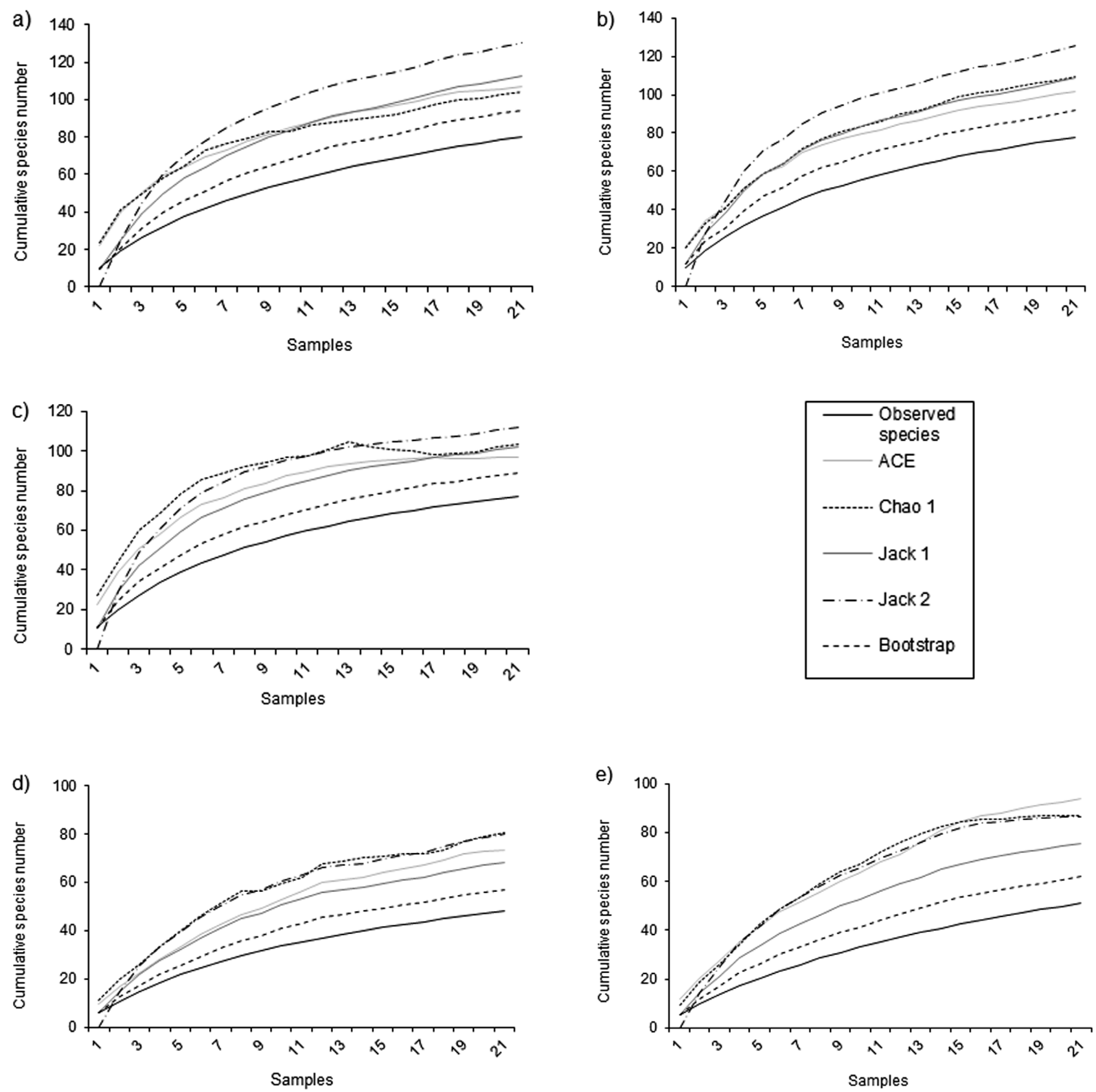

Fig. 5. Observed and estimated species accumulation curves for scuttle flies as a function of the cumulative number of samples (based on 100 randomizations) in the five plots studied: mesohumid beech forest (a), humid beech forest (b), dry beech forest (c), Juniperus scrubland (d) and Calluna scrubland (e). The ICE and Chao 2 estimators are not included as they are unstable and do not result in asymptotic curves.

Table 3. Estimated species richness quantified using different estimators for the five plots studied.

\begin{tabular}{|c|c|c|c|c|c|c|c|c|c|c|}
\hline & \multicolumn{2}{|c|}{ Mbf } & \multicolumn{2}{|c|}{$\mathrm{Hbf}$} & \multicolumn{2}{|c|}{ Dbf } & \multicolumn{2}{|c|}{ Jsc } & \multicolumn{2}{|c|}{ Csc } \\
\hline & $S$ & $\%$ & $S$ & $\%$ & $S$ & $\%$ & $S$ & $\%$ & $S$ & $\%$ \\
\hline $\begin{array}{l}\text { Observed } \\
\text { Individuals }\end{array}$ & $\begin{array}{c}80 \\
730\end{array}$ & & $\begin{array}{c}78 \\
843\end{array}$ & & $\begin{array}{c}77 \\
1.376\end{array}$ & & $\begin{array}{c}48 \\
418\end{array}$ & & $\begin{array}{c}51 \\
317\end{array}$ & \\
\hline Singletons & 26 & $32.50 \%$ & 25 & $32.05 \%$ & 24 & $31.16 \%$ & 18 & $37.50 \%$ & 24 & $47.05 \%$ \\
\hline $\mathrm{ACE}$ & 106.81 & $74.90 \%$ & 101.64 & $76.74 \%$ & 96.69 & $79.64 \%$ & 73.39 & $65.40 \%$ & 94.11 & $54.19 \%$ \\
\hline ICE & 122.84 & $65.13 \%$ & 112.25 & $69.49 \%$ & 102.19 & $75.35 \%$ & 74.09 & $64.79 \%$ & 92.25 & $55.28 \%$ \\
\hline Chao 1 & 104.14 & $76.82 \%$ & 109.25 & $71.40 \%$ & 103.18 & $74.63 \%$ & 80.4 & $59.70 \%$ & 87 & $58.62 \%$ \\
\hline Chao 2 & 118.53 & $67.49 \%$ & 114.57 & $68.08 \%$ & 98.13 & $78.47 \%$ & 75.56 & $63.53 \%$ & 73.53 & $69.36 \%$ \\
\hline Jack 1 & 112.38 & $71.19 \%$ & 108.48 & $71.90 \%$ & 101.76 & $75.67 \%$ & 68 & $70.59 \%$ & 75.76 & $67.32 \%$ \\
\hline Jack 2 & 130.25 & $61.42 \%$ & 125.4 & $62.20 \%$ & 111.53 & $69.04 \%$ & 80.12 & $59.91 \%$ & 86.39 & $59.03 \%$ \\
\hline Bootstrap & 94.58 & $84.58 \%$ & 91.7 & $85.06 \%$ & 88.9 & $86.61 \%$ & 56.92 & $84.33 \%$ & 62.28 & $81.89 \%$ \\
\hline
\end{tabular}

Number of observed and estimated species $(S)$ and percentage of species captured and singletons compared to the estimated number of species (\%). Mbf - mesohumid beech forest, $\mathrm{Hbf}$ - humid beech forest, Dbf - dry beech forest, Jsc - Juniperus scrubland, Csc - Calluna scrubland. 


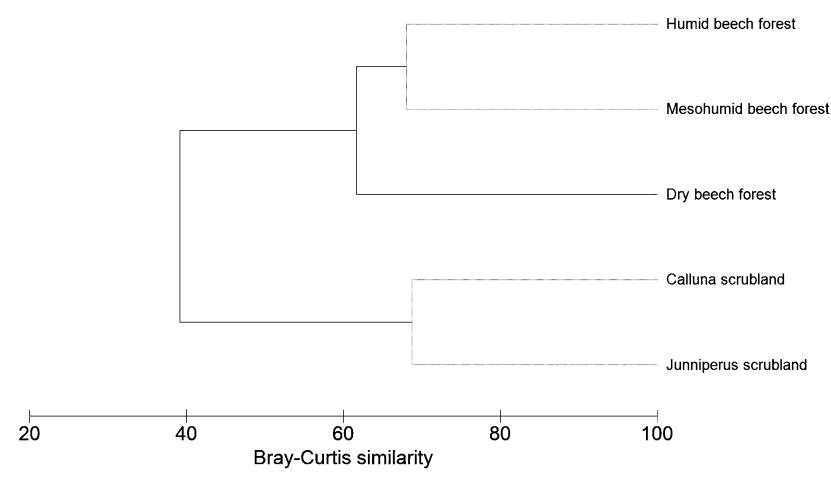

Fig. 6. Cluster analysis of sites using the Bray-Curtis similarity index. Dotted line indicate clusters that were significantly similar in the SIMPROF analysis.

higher in scrubland than beech forest. Dbf plot had the lowest evenness (Table 4).

\section{$\beta$-diversity}

The cluster analysis separated plots into two groups, the first included the plots in the beech forest and the second those in scrubland. However, the Dbf plot was separated from the other two beech forest plots by the SIMPROF test (Fig. 6). The PERMANOVA analysis of Bray-Curtis similarity revealed significant differences between both habitats $(p=0.0001)$ and homogeneous dispersion $(p=0.63)$, supporting the results of the Cluster analysis.

According to the SIMPER analyses, the species that contributed most to the similarity of the different plots in beech forest were Megaselia pusilla, M. diversa, M. flavicans, M. longicostalis, M. lata, M. angusta and Diplonevra florescens (Table 5). The two-dominant species in this habitat, $M$. pectoralis and $M$. subpleuralis, were not included on this list because their abundances in the plots were very different.

The species that contributed most to the similarity between the two plots in scrubland were Megaselia pusilla, $M$. pumila, $M$. diversa, $M$. brevicostalis, $M$. longicostalis, Metopina galeata and Diplonevra nitidula (Table 6). The species that contributed most to the differences in species composition of the scuttle fly communities in the two habitats, were Megaselia pumila, M. brevicostalis, Triphleba opaca and Metopina galeata, which are dominant in scrubland, and Diplonevra florescens, M. lata, M. flavicans and M. subtumida, which are dominant in beech forest (Table 7).

\section{DISCUSSION}

The structure of scuttle fly communities were significantly different in the beech forest and scrubland studied.

Table 4. Summary values of species richness (S), number of individuals $(N)$, Shannon index $\left(H^{\prime}\right)$ and evenness $\left(J^{\prime}\right)$ of 16 fortnightly samples for the five plots studied.

\begin{tabular}{lcccc}
\hline Plot & $S$ & $N$ & $H^{\prime}$ & $J^{\prime}$ \\
\hline Mesohumid beech forest & 78 & 728 & 3.20 & 0.73 \\
Humid beech forest & 79 & 844 & 3.03 & 0.69 \\
Dry beech forest & 75 & 1351 & 2.55 & 0.59 \\
Juniperus scrubland & 47 & 415 & 2.98 & 0.77 \\
Calluna scrubland & 50 & 310 & 3.08 & 0.79 \\
\hline
\end{tabular}

Table 5. Results of SIMPER analysis of the beech forest plots. The species are ordered based on the percentage they contribute to average similarity.

\begin{tabular}{lcccc}
\hline Beech forest species & $\begin{array}{c}\text { Avg. } \\
\text { Abund }\end{array}$ & $\begin{array}{c}\text { Avg. } \\
\text { Simil }\end{array}$ & $\begin{array}{c}\% \\
\text { Contribution }\end{array}$ & $\begin{array}{c}\% \\
\text { Cumulated }\end{array}$ \\
\hline Megaselia pusilla & 6.26 & 6.67 & 10.09 & 10.09 \\
Megaselia diversa & 6.10 & 6.54 & 9.89 & 19.99 \\
Megaselia flavicans & 4.33 & 4.49 & 6.79 & 26.78 \\
Megaselia longicostalis & 4.73 & 4.19 & 6.33 & 33.11 \\
Megaselia lata & 4.27 & 4.03 & 6.10 & 39.21 \\
Megaselia angusta & 3.94 & 3.45 & 5.21 & 44.43 \\
Diplonevra florescens & 4.98 & 2.90 & 4.39 & 48.82 \\
Megaselia subtumida & 2.61 & 2.55 & 3.85 & 52.67 \\
Megaselia pleuralis & 2.72 & 2.36 & 3.57 & 56.24 \\
Megaselia scutellaris & 3.02 & 2.13 & 3.23 & 59.47 \\
Megaselia ruficornis & 2.06 & 2.04 & 3.09 & 62.56 \\
\hline
\end{tabular}

Avg. Abund - square-root transformed average abundance; Avg Simil - average Bray-Curtis similarity between pairs of plots; \% Contribution - percentage contribution of each species to similarity between plots; \% Cumulated - cumulated percentage of contribution (cut-off percentage: $60 \%$ ).

In accordance with our hypothesis, the percentage, density and species richness of scuttle flies was higher in beech forest than scrubland. In contrast, evenness was lower in beech forest than in scrubland due to the high dominance of a few specialised saprophagous species, while generalist species dominated in scrubland. These differences in scuttle fly communities can be attributed to differences in vegetation and microclimatic in the two habitats. Because the scrubland is at a higher altitude than the beech forest it was more windy and colder there than the beech forest (Rodà, 1983; Boada, 2001). Similarly, deciduous forests with acid soil usually have a thicker soil litter layer with more decaying plant matter than scrubland, which favours Diptera with saprophagous larvae (Terrades, 1984; Hövemeyer, 1991). The saprophagous Megaselia pectoralis, Megaselia diversa, M. pectorella and Diplonevra florescens contributed most to the dominance of this trophic group in the beech forest. The thin litter layer together with a higher species richness of herbaceous plants and shrubs in scrubland relative to beech forest (Bolòs, 1983; Boada, 2001) may provide a greater diversity of resources for scuttle flies. This might account for the higher abundance of generalists and evenness of trophic groups in scrubland than beech forest. The dominant species in scrubland were the polyphagous $M$. pusilla and mycophagous M. pumila,

Table 6. Results of SIMPER analysis of the scrubland plots. The species are ordered based on their percentage contribution to average similarity.

\begin{tabular}{lcccc}
\hline Scrublands species & $\begin{array}{c}\text { Avg. } \\
\text { Abund }\end{array}$ & $\begin{array}{c}\text { Avg. } \\
\text { Simil }\end{array}$ & $\begin{array}{c}\% \\
\text { Contribution }\end{array}$ & $\begin{array}{c}\% \\
\text { Cumulated }\end{array}$ \\
\hline Megaselia pusilla & 8.04 & 11.36 & 16.04 & 16.04 \\
Megaselia pumila & 7.10 & 11.24 & 15.87 & 31.90 \\
Megaselia diversa & 5.24 & 8.29 & 11.70 & 43.60 \\
Megaselia brevicostalis & 4.23 & 6.42 & 9.06 & 52.66 \\
Megaselia longicostalis & 3.52 & 5.24 & 7.40 & 60.06 \\
Metopina galeata & 3.35 & 4.06 & 5.73 & 65.79 \\
Diplonevra nitidula & 3.67 & 3.71 & 5.23 & 71.02 \\
\hline
\end{tabular}

Avg. Abund - square-root transformed average abundance; Avg. Simil - average Bray-Curtis similarity between pairs of plots. \% Contribution - percentage contribution of each species to similarity between plots. \% Cumulated - cumulated percentage of contribution (cut-off percentage: $70 \%$ ). 
Table 7. Results of SIMPER analysis of beech forest and scrubland plots. The species are ordered based on their percentage contribution to average dissimilarity between habitats.

\begin{tabular}{|c|c|c|c|c|c|}
\hline Species & $\begin{array}{c}\text { Beech forest } \\
\text { Av. Abund }\end{array}$ & $\begin{array}{l}\text { Scrubland } \\
\text { Av. Abund }\end{array}$ & Av. Dissimil & $\%$ Contribution & $\%$ Cumulated \\
\hline Megaselia pumila & 1.93 & 7.1 & 3.46 & 6.33 & 6.33 \\
\hline Diplonevra florescens & 4.98 & 0.5 & 2.95 & 5.39 & 11.73 \\
\hline Megaselia lata & 4.27 & 0 & 2.87 & 5.24 & 16.97 \\
\hline Megaselia flavicans & 4.33 & 0.5 & 2.55 & 4.67 & 21.64 \\
\hline Megaselia brevicostalis & 1.14 & 4.23 & 2.08 & 3.81 & 25.44 \\
\hline Megaselia subtumida & 2.61 & 0 & 1.74 & 3.19 & 28.63 \\
\hline Triphleba opaca & 0.82 & 2.99 & 1.63 & 2.98 & 31.61 \\
\hline Metopina galeata & 0.94 & 3.35 & 1.58 & 2.89 & 34.50 \\
\hline Megaselia giraudii & 2.09 & 0 & 1.39 & 2.54 & 37.04 \\
\hline Megaselia pleuralis & 2.72 & 0.71 & 1.38 & 2.52 & 39.56 \\
\hline Megaselia ruficornis & 2.06 & 0 & 1.38 & 2.52 & 42.08 \\
\hline Megaselia mallochi & 0.33 & 2.3 & 1.28 & 2.34 & 44.42 \\
\hline Megaselia angusta & 3.94 & 2.29 & 1.27 & 2.32 & 46.74 \\
\hline Diplonevra nitidula & 3.15 & 3.67 & 1.2 & 2.20 & 48.94 \\
\hline Megaselia pusilla & 6.26 & 8.04 & 1.16 & 2.11 & 51.05 \\
\hline Megaselia subfuscipes & 2.25 & 1.32 & 1.12 & 2.04 & 53.09 \\
\hline Conicera floricola & 1.63 & 0 & 1.07 & 1.96 & 55.05 \\
\hline Diplonevra funebris & 0 & 1.57 & 1.06 & 1.94 & 56.98 \\
\hline Megaselia nigra & 1.87 & 1 & 1.04 & 1.91 & 58.89 \\
\hline Megaselia scutellaris & 3.02 & 1.57 & 1 & 1.82 & 60.72 \\
\hline
\end{tabular}

Av. Abund - square-root transformed average abundance; Av. Dissimil - average Bray-Curtis dissimilarity between pairs of plots in different habitats; \% Contribution - percentage contribution of each species to dissimilarity between plots. \% Cumulated: cumulated percentage of contribution (cut-off percentage: $60 \%$ ).

while the abundance of saprophagous species was lower in beech forest (Fig. 3). Mycophagous species are also more frequent in clear-cuts than in old-growth pine forest (Durska, 2013). Finally, the frequency of flower-visiting species (M. pumila, $M$ breviscostalis, D. nitidula, M. longicostalis, Triphleba opaca and M. xanthozona) (Disney, 1994) was higher in scrubland than beech forest, which is most likely related to the higher abundance and diversity of insect-pollinated plants in scrubland than beech forest (Bolòs, 1983; Boada, 2001).

Overall, scuttle fly species composition was significantly different in the habitats, but was similar among plots within the same habitat. However, interestingly, the Dbf plot had higher abundance and lower evenness than the more humid beech forest plots. These differences are most likely due to the higher cover of herbaceous plants in Dbf than in the more humid beech plots. A higher cover of herbaceous plants might enhance the conditions for egg laying or sheltering and overwintering for Diptera. A slightly positive effect of the herbaceous plant cover on scuttle fly relative abundance is also reported for other European beech forests (Scherber et al., 2014).

The percentage of scuttle flies relative to other Diptera in the Montseny beech forest was $8.1 \%$, which is similar or higher than the values reported for other European beech forests on acid soil in which scuttle flies were sampled using emergence traps (Altmüller, 1976, 1979; Hövemeyer, 1984, 1991; Ascaso, 1989; Buck, 1994). Notably, the percentage of scuttle flies in beech forest in which water traps and suction samples were used (Scherber et al., 2014) was higher (25\%) than in the Montseny beech forest. This indicates that the percentage of scuttle flies recorded depends on the sampling method used. Likewise, the percentage of scuttle flies in the Montseny beech forest was in general higher than in European coniferous forests (Mollon, 1982;
Olechowicz, 1984, 1988; Hövemeyer, 1991), but lower than in riverine forest (Buchs, 1983, 1988; Buck, 1997).

In relation to the Montseny scrubland, the percentage of scuttle flies relative to other Diptera (5.1\%) was lower than that reported for other open-area habitats. Most studies on scuttle flies in open-area habitats have been done in crops and meadows (Hövemeyer, 1991; Buck, 1994, 1997; Weber \& Prescher, 1995). These habitats are rich in decayed soil organic matter and some are routinely fertilized, which can increase the abundance of saprophagous larvae.

Species dominance structure differed in the two habitats. It also seems to differ from that reported for other similar European habitats. On the one hand, Megaselia pectoralis and M. subpleuralis, which were the dominant species in the Montseny beech forest, have low relative abundances in other European forests (Durska, 1996, 2001, 2009, 2013; Prescher et al., 2002; Brenner, 2005, 2008; Durska et al., 2010; Bonet et al., 2011; Scherber et al., 2014), Similarly, Megaselia diversa, M. pectorella and Diplonevra florescens, which were subdominant in the beech forest, have low relative abundances in other European forests (Durska, 1996, 2009, 2013; Buck, 1997; Weber \& Schiegg, 2001; Prescher et al., 2002; Brenner, 2005; Durska et al., 2010; Zmihorski \& Durska, 2010; Bonet et al., 2011; Scherber et al., 2014; Disney, 2015), except for a few coniferous forests where these species are dominant or subdominant (Durska, 2001, 2013; Brenner, 2002; Durska et al., 2010). The rest of the subdominant species in the Montseny beech forest (M. pusilla, M. longicostalis, M. basispinata and M. superciliata) are usually rare in other European forest (Durska, 1996, 2001, 2013; Durska et al., 2010; Zmihorski \& Durska, 2010; Bonet et al., 2011; Disney, 2015). On the other hand, the dominant species in scrubland, Megaselia pusilla, M. pumila, M. diversa and M. superciliata, are subdominant or accessory in other open-area habitats in Europe (Disney et al., 1981b; Prescher et al., 2000; Durska, 2001, 
2006, 2013; Brenner, 2003), except for M. pusilla, which it is also dominant in pastures and crops in Switzerland and England (Disney et al., 1981b; Prescher et al., 2000). $M$. brevicostalis and Triphleba opaca, which are characteristic of open-area habitats and colonists of disturbed areas resulting from clear-cutting or wildfires in forests (Durska et al., 2010; Durska, 2013, 2015) were subdominant in scrubland and rare in beech forest. M. brevicostalis is recorded in very different habitats, such as wheat and alfalfa fields (Disney, 1994), meadows, pine, spruce, chestnut and riparian forests, and also in saline habitats (Prescher, 1992; Durska, 2001, 2009; Prescher et al., 2002; Durska et al., 2005; Bonet et al., 2011). The rest of the subdominant species in scrubland, are accessory or absent in other open-area habitats in Europe (Disney et al., 1981b; Prescher et al., 2000; Brenner, 2003, 2005, 2008; Durska et al., 2010), except for Diplonevra nitidula that is dominant or subdominant in grasslands and crops (Disney, 1980; Disney et al., 1981b; Prescher, 1992; Buck, 1994).

The efficiency of the sampling effort was higher in beech forest than scrubland. The range of inventory completeness (i.e. ratio of the observed richness and Chao 1) for the Montseny beech forest plots was higher (71-77\%) than that recorded in mixed coniferous and deciduous forests in Sweden (60-67\%) for Megaselia species and none of the estimators were asymptotic (Bonet et al., 2011). The different sampling method used (Malaise traps) in the survey of Bonet et al. (2011) could explain the lower inventory completeness. In contrast, the range of inventory completeness for the Montseny scrubland plots was lower (58-60\%) than that recorded for open-area habitats after wildfires (72$81 \%$ ), for which there are asymptotic curves (Bonet et al., 2011). Differences among studies may be explained by the fact that the wildfire areas sampled by Bonet et al. (2011) were at different sites, each with its own species composition. In addition, the high amount of dead wood after a wildfire could have increased the soil-inhabiting species richness, making the use of Malaise traps more effective.

In conclusion, Mediterranean mountain beech forest and scrubland differ in their scuttle fly community structure. Percentage, density and species richness of scuttle flies was higher in beech forest than scrubland, while evenness was lower in beech forest than scrubland due to higher dominance of generalist species in scrubland and the specialized saprophagous species in beech forest. These differences support the idea that the Phoridae are a suitable bioindicator for comparative and evaluative surveys of ecologically distinct habitats. Future studies should assess other habitats at lower altitudes in the Montseny Natural Park in order to determine the distribution of scuttle fly species, especially in holm oak forest, which is the largest habitat in the area. Specifically, it would be interesting to know if the generalists M. pusilla and M. longicostalis, which have polyphagous larvae and are widely distributed in the beech forest and highland scrubland studied, are also present in other habitats in the Natural Park.

ACKNOWLEDGEMENTS. We acknowledge the Diputación de Barcelona for supporting this entomological project at the Mont- seny Natural Park in 1990-1991. We would like to thank J. Pinyol for their help and advice with multivariate statistical programs and comments on our work. We also acknowledge P. Villar-Salvador for reviewing the English and C. Bach for lending us the LEICA microscope.

\section{REFERENCES}

AltMÜLleR R. 1976: Ökoenergetische Untersuchungen an Dipterenpopulationen im Buchenwald (Luzulo-Fagetum). - Verh. Ges. Ökol. 5: 133-138.

AltMÜLLER R. 1979: Untersuchungen über den Energieumsatz von Dipterenpopulationen im Buchenwald (Luzulo-Fagetum). - Pedobiologia 19: 245-278.

ANDERSON M.J. 2001: A new method for non-parametric multivariate analysis of variance. - Austral Ecol. 26: 32-46.

Anderson M.J., Gorley R.N. \& Clarke K.R. 2008: PERMANOVA + for PRIMER: Guide to Software and Statistical Methods. PRIMER-E, Plymouth, UK.

AsCaso C. 1989: Producción secundaria de artrópodos estimada mediante trampas de emergencia en dos bosques del Montseny (Barcelona). - Orsis 4: 81-97.

Bächlı G. 1970: Der Waldboden als Entwicklungssubstrat von Dipteren. - Vierteljahrsschr. Naturf. Ges. Zürich 115: 255259.

BäHRMANN R. 1984: Die Zweifluger der Kraut- und Strauchschicht des Leutratals bei Jena (Thüringen) - ein ökofaunistischer Vergleich. - Zool. Jahrb. Abt. Syst. 111: 175-217.

Begon M., Townsend C.R. \& Harper J.L. 2008: Ecology. From Individuals to Ecosystems. Blackwell, Oxford, $738 \mathrm{pp}$.

Boada M. 2001: Manifestacions del canvi ambiental global al Montseny. PhD Thesis, Universidad Autónoma de Barcelona, Bellaterra, $430 \mathrm{pp}$.

Boada M. \& Ullastres H. 1998: El macizo del Montseny, guía para visitarlo. Brau Ediciones del Bronce, Figueres, 110 pp.

Bolòs O. 1983: La Vegetació del Montseny. Diputació, Servei de Parcs Naturals, Barcelona, 170 pp.

Bonet J., Ulefors S.-O., Viklund B. \& Pape T. 2011: Species richness estimations of the megadiverse scuttle fly genus Megaselia (Diptera: Phoridae) in a wildfire-affected hemiboreal forest. - Insect Sci. 18: 325-348.

BRASSE D. 1975: Die Arthropodenfauna von Getreidefeldern auf verschiedenen Böden im Braunschweiger Raum. - Pedobiologia 15: 405-414.

Brenner S. 2002: Buckelfliegen (Diptera: Phoridae) an der Waldgrenze bei Innsbruck (Nordtirol, Österreich). - Ber. Nat.Med. Ver. Innsbruck 89: 237-248.

BRENNER S. 2003: Tageszeitliche Flugaktivität von Buckelfliegen (Insecta: Diptera: Phoridae) in der alpinen Stufe der Ötztaler Alpen (Österreich). — Ber. Nat.-Med. Ver. Innsbruck 90: 207-218.

BREnNER S. 2004: Scuttle flies (Diptera, Phoridae) from a riverine forest in South Tyrol (Italy). — Gredleriana 4: 295-304.

BRENNER S. 2005: Buckelfliegen (Insecta, Diptera: Phoridae) aus dem Naturschutzgebiet Antelsberg bei Tarrens, cinem inneralpinen Wärmestandort (Nordtirol, Österreich). — Linzer Biol. Beitr. 37: 223-234.

BRENNER S. 2008: New records of scuttle flies (Diptera: Brachychera, Phoridae) from the nature reserve Schlern - Rosengarten (South Tyrol, Italy). — Gredleriana 8: 411-427.

Büchs W. 1983: Jahresperiodische Aktivität und Wechselbeziehungen von Arthropoden aus der Sammregion eines harholzauenwaldes (Fraxino-Ulmetum). - Verh. Dtsch. Zool. Ges. 1983: 210 . 
Büchs W. 1988: Stamm-und Rinden-zoozönosen verschiedener Baumarten des Hartholzauenwaldes und ihr Indikatorwert für die Früherkennung von Baumschäden. PhD Thesis, Rheinische Friederich-Wilhelms-Universität, 813 pp.

BucK M. 1994: Sphaeroceridae and Phoridae (Diptera) collected by emergence traps from various terrestrial habitats in Southern Germany. - Stud. Dipterol. 1: 93-106.

BucK M. 1997: Untersuchungen zur ökologischen Einnischung saprophager Dipteren unter besonderer Berücksichtigung der Phoridae und Sphaeroceridae (Brachycera/Cyclorrhapha). PhD Thesis, University of Ulm, $194 \mathrm{pp}$.

CARles-Tolrá M. 1995: Dípters. In Barrientos J.A. (ed.): El patrimoni biològic del Montseny. Catàlegs de fauna i flora, 2. Diputació de Barcelona, Servei de Parcs Naturals, pp. 69-85.

Chazdon R.L., Colwell R.K., Denslow J.S. \& Guariguata M.R. 1998: Statistical methods for estimating species richness of woody regeneration in primary and secondary rain forests of northeastern Costa Rica. In Dallmeier F. \& Comiskey J.A (eds): Forest Biodiversity Research, Monitoring and Modeling. Conceptual Background and Old World Case Studies. Parthenon, Carnforth, pp. 285-309.

Clarke K.R. \& Gorley R.N. 2006: PRIMER Ver. 6. User Manual/Tutorial. PRIMER-E, Plymouth, UK.

Colwell R.K. 2009: EstimateS: Statistical Estimation of Species Richness and Shared Species from Samples. Ver. 8.2. User's Guide and Application. URL: http://purl.oclc.org/estimates.

Colwell R.K. \& Coddington J.A. 1994: Estimating terrestrial biodiversity through extrapolation. - Phil. Trans. R. Soc. (B) 345: 101-118.

Colwell R.K., Mao C.X. \& Chang J. 2004: Interpolating, extrapolating, and comparing incidence-based species accumulation curves. - Ecology 85: 2717-2727.

DisNey R.H.L. 1980: Some soil-inhabiting scuttle flies. — Entomol. Mon. Mag. 115: 231-232.

Disney R.H.L. 1990: Some myths and the reality of scuttle fly biology. - Antenna 14: 64-67.

Disney R.H.L. 1994: Scuttle Flies: The Phoridae. Chapman \& Hall, London, 467 pp.

DisNey R.H.L. 2015: Scuttle flies (Diptera, Phoridae) from the canopies of oak trees (Fagaceae) in Norway, including 13 new species. - Nor. J. Entomol. 62: 20-52.

Disney R.H.L., Coulson J.C. \& Butterfield J. 1981a: A survey of a scuttle flies of upland habitats in Northern England. Naturalist 106: 53-66.

Disney R.H.L., Henderson I.F., Perry J.N. \& Clements R.O. 1981b: Phoridae from English Pasture soils. - Pedobiologia 22: $366-378$

Disney R.H.L., Prescher S. \& Ashmole N.P. 2010: Scuttle flies (Diptera: Phoridae) of the Canary Islands. - J. Nat. Hist. 44: 107-218.

DURSKA E. 1981: Phoridae (Diptera) of Warsaw. - Memorab. Zool. (Warszawa) 35: 47-56.

DURSKA E. 1996: The species composition and structure of scuttle fly communities (Diptera, Phoridae) in mature tree stands in pine forests at different stages of habitat degradation. Fragm. Faun. 39: 267-285.

DURSKA E. 2001: Secondary succession of scuttle fly communities (Diptera: Phoridae) in moist pine forest in Bialowieza Forest. - Fragm. Faun. 44: 79-128.

DuRSKA E. 2006: Diversity of the scuttle fly (Diptera: Phoridae) communities in the plantations of moist pine forests of the Bialowieza Primeval Forest and the Tuchola Forest (Poland). Biodiv. Conserv. 15: 385-393.
DurSKa E. 2009: The scuttle fly (Diptera: Phoridae) assemblages of pine plantations of the Biala Forest (Poland). - Entomol. Fenn. 20: $170-178$.

DURSKA E. 2013: Effects of disturbances on scuttle flies (Diptera: Phoridae) in pine forests. - Biodiv. Conserv. 22: 1991-2021.

DuRsKa E. 2015: Effects of fire on scuttle flies (Diptera: Phoridae) in a pine forest in Poland. - Entomol. Fenn. 26: 181-193.

Durska E., Kaczorowska E. \& Disney R.H.L. 2005: Scuttle flies (Diptera: Phoridae) of saline habitats of the Gulf of Gdansk, Poland. - Entomol. Fenn. 16: 159-164.

Durska E., Bonet J. \& VikLund B. 2010: The scuttle fly (Diptera: Phoridae) assemblages of a wildfire-affected hemiboreal old-growth forest in Tyresta (Sweden). - Entomol. Fenn. 21: 19-32.

García-Romera C. \& Barrientos J.A. 2014a: La fauna de Phoridae (Diptera) en el Parque Natural del Montseny (Cataluña, España). Citas nuevas para la Península Ibérica. - Bol. Soc. Entomol. Aragon. 54: 237-261.

García-Romera C. \& Barrientos J.A. 2014b: Nine new species of Phoridae (Insecta: Diptera) from Spain. - Zootaxa 3857: 244-260.

Goos M. 1975: Influence of aphicides used in sugar-beet plantations on arthropods. IV. Studies on the flying insects captured in yellow receptacles. - Pol. Pis. Entomol. 45: 423-444.

HöVEMEYER K. 1984: Die Dipterengemeinschaft eines Buchenwaldes auf Kalkgestein: Produktion an Imagines, Abundanz und räumliche Verteilung insbesondere der larven. - Pedobiologia 26: 1-15.

HÖVEMEYER K. 1991: The study of dipterous populations and communities in european terrestrial ecosystems. In Weismann L., Orszagh I. \& Pont A.C. (eds): Proceedings of the Second International Congress of Dipterology Bratislava, Czechoslovakia, 1990. SPB Academic Publishing, The Hague, pp. 99-109.

MagurRan A.E. 2004: Measuring Biological Diversity. Blackwell, Oxford, $256 \mathrm{pp}$.

Mollon A. 1982: Etude d'un écosystème forestier mixte. VII. Composition et phénologie du peuplement des Dipterès à larves édaphiques. - Rev. Ecol. Biol. Sol 19: 289-306.

MoRENo C.E. 2001: Métodos para medir la biodiversidad. M\&T - Manuales y Tesis SEA, Vol. 1. Zaragoza, 83 pp.

Olechowicz E. 1971: Productivity investigation of two types of meadows in the Vistula valley VIII. The number of emerged Diptera and their elimination. - Ekol. Pol. 19: 183-195.

Olechowicz E. 1984: Herb layer entomofauna of different forest ecosystems in the Kampinos forest and its participation in cycling of elements. - Ekol. Pol. 32: 43-60.

Olechowicz E. 1988: The structure of community of Diptera imagines of different forest ecosystems in the Kampinos forest. Ekol. Pol. 36: 509-529.

Prescher S. 1992: Ökologie und Biologie der Diptera, insbesondere der Brachycera, eines klärschlamm gedüngten Ackerbodens. PhD Thesis, Der Technischen Universität Carolo-Wilhelmina zu Braunschweig.

Prescher S. \& Haenni J.P. 2001: Some scuttle flies (Diptera: Phoridae) from the Swiss Jura. - Bull. Soc. Neuchâtel. Sci. Nat. 124: 125-130.

Prescher S., Obrist M.K. \& Duelli P. 2000: Die Phoridenfauna (Diptera, Brachycera) naturnaher Biotope und intensiv genutzter kulturflächen im Schweizer Mittelland. - Mitt. Schweiz. Entomol. Ges. 73: 265-275.

Prescher S., Moretti M. \& Duelli P. 2002: Scuttle flies (Diptera, Phoridae) in Castanea sativa forests in the Southern Alps (Ticino, Switzerland), with thirteen species new to Switzerland. - Bull. Soc. Entomol. Suisse 75: 289-298. 
Putman R.J. 1994: Community Ecology. Chapman \& Hall, London, $178 \mathrm{pp}$.

Ricou G. 1978: The dipteran community of a newly sown grassland. - Sci. Proc. R. Dublin Soc. (A) 6: 259-271.

RodÀ F. 1983: Biogeoquímica de les aigües de pluja i de drenatge en alguns ecosistemes forestals del Montseny. PhD Thesis, Universitat Autònoma de Barcelona, 137 pp.

Ryder C., Moran J., Mc Donnell R. \& Gormally M. 2005: Conservation implications of grazing practices on the plant and dipteran communities of a turlough in Co. Mayo, Ireland. Biodivers. Conserv. 14: 187-204.

Scherber C., Vockenhuber E.A., Stark A., Meyer H. \& TsCHARNTKE T. 2014: Effects of tree and herb biodiversity on Diptera, a hyperdiverse insect order. — Oecologia 174: 13871400.

Sokal R.R. \& Rohlf F.J. 1995: Biometry: The Principles and Practice of Statistics in Biological Research. Freeman, New York, $887 \mathrm{pp}$.

SzADZIEwSKa M.M. 1977: Geofilne muchówki (Diptera) rezerwatu 'Las Piwnicki' kolo Torunia. - Acta Univ. Nicolai Copernici Biol. 19: 107-115.
TERRADES J. 1984: Introducció a l'ecologia del faig del Montseny. Diputació de Barcelona, Servei de Parcs Naturals, 83 pp.

Weber G. \& Prescher S. 1990: Studies on the ecology of Phoridae (Diptera). - Pedobiologia 34: 183-189.

Weber G. \& Prescher S. 1995: Die Mücken und Fliegen eines klärschlammgedüngten Ackers. In Nentwig W. \& Poehling H.-M. (eds): Agrarökologie 15. Paul Haupt, Bern, Stuttgart, Wien, pp. 5-100.

Weber G. \& Schiegg K. 2001: Scuttle flies (Diptera: Phoridae) from the forest reserve Sihlwald ZH. - Stud. Dipterol. 8: 271-276.

Weber G., Prescher S., Ulefors S.-O. \& Viklund B. 2006: Fifty-eight species of scuttle flies (Diptera: Phoridae: Megaselia spp.) new to Sweden from the Tyrestra National Park and Nature Reserve. - Stud. Dipterol. 13: 231-240.

ZMIHORSKI M. \& DURSKA E. 2010: The effect of contrasting management types on two distinct taxonomic groups in a largescaled windthrow. - Eur. J. For. Res. 130: 589-600.

Received November 2, 2015; revised and accepted February 2, 2017 Published online April 11, 2017 